\title{
Optical coherence tomography angiography (OCTA) findings in Serpiginous Choroiditis
}

Sergio Macedo* ${ }^{*}$, Dominika Pohlmann, Matthias Lenglinger, Uwe Pleyer, Antonia M. Joussen and Sibylle Winterhalter

\begin{abstract}
Background: To describe changes in the retina/choroid in patients with Serpiginous Choroiditis (SC) by Optical Coherence Tomography Angiography (OCTA) in a multimodal imaging approach.

Methods: Prospective, monocentric study of 24 eyes of 12 consenting patients diagnosed with SC, who underwent OCTA, which was analyzed and compared to other methods such as enhanced depth imaging-OCT, fluorescein angiography, indocyanine green angiography, and fundus autofluorescence.

Results: The study group consisted of 9 patients with peripapillary SC, 1 macular SC, and 2 atypical cases. All eyes presented an inactive SC confirmed by standard imaging. OCTA demonstrated the lesions tridimensionally in great detail. There was no difference in the angioarchitecture among the 3 forms of SC. A loss of the choriocapillaris/retinal pigment epithelium left a "window-defect", where the vessels of larger caliber of the choroid became recognizable and their appearance inverted ("white-on-black"). A relationship between the presence of segmentation errors (SE) in the slabs and low visual acuity was established with a one-way ANOVA.

Conclusions: OCTA was able to non-invasively assess vascular lesions of the choroid/retina in patients with SC with a high degree of correlation to other diagnostic modalities. Consequent long-term assessments could lead to a better understanding of disease progression.
\end{abstract}

Keywords: Fluorescein angiography, Fundus autofluorescence, Indocyanin green angiography, Optical coherence tomography angiography, Posterior uveitis, Serpiginous Choroiditis

\section{Background}

Serpiginous choroiditis (SC) is a recurrent inflammatory primary choriocapillaropathy, belonging to a group of rare idiopathic diseases called "white-dot syndromes", which were first described by Ezra in 1995 [1]. The disease commonly occurs bilaterally and asymmetrically, progressing in a centrifugal and phased manner. Patients, mostly

\footnotetext{
* Correspondence: sergio.macedo@charite.de

Department of Ophthalmology, Campus Virchow- Klinikum, Charité University Medicine Berlin, corporate member of Freie Universität Berlin, Humboldt- Universität zu Berlin and Berlin Institute of Health, Augustenburger Platz 1, 13353 Berlin, Germany
}

between the age of 20 and 60 years, usually complain of blurry vision, scotomas, and metamorphopsia, equating to a diminished quality of life with irreversible loss of vision in cases where the macula is affected [2,3].

Three types of SC are currently recognized depending on the primary location of inflammation $[4,5]$. The "peripapillary form" accounts for approximately $80 \%$ of cases and begins near the optic disc with a late affection of the macular region, whereas the "macular form" has a poor initial visual prognosis because of the early disease manifestation of the macula. In contrast to this, the "atypical form" starts in the periphery in a partly

(c) The Author(s). 2020 Open Access This article is licensed under a Creative Commons Attribution 4.0 International License, which permits use, sharing, adaptation, distribution and reproduction in any medium or format, as long as you give appropriate credit to the original author(s) and the source, provide a link to the Creative Commons licence, and indicate if changes were made. The images or other third party material in this article are included in the article's Creative Commons licence, unless indicated otherwise in a credit line to the material. If material is not included in the article's Creative Commons licence and your intended use is not permitted by statutory regulation or exceeds the permitted use, you will need to obtain permission directly from the copyright holder. To view a copy of this licence, visit http://creativecommons.org/licenses/by/4.0/ The Creative Commons Public Domain Dedication waiver (http://creativecommons.org/publicdomain/zero/1.0/) applies to the data made available in this article, unless otherwise stated in a credit line to the data. 
multifocal manner, may reach the macular region and possibly initiates as acute posterior multifocal placoid pigment epitheliopathy [6]. Before the diagnosis of SC can be determined, tuberculosis related uveitis has to be excluded [7].

There is currently no consensus regarding its optimal treatment options, which consists mostly of immunosuppressive substances and biologicals $[8,9]$. However, disease recurrence is often observed despite therapy. A better anticipation of drug response and disease progression could help lower treatment burden.

In the past years, a multimodal imaging approach has been described to facilitate diagnosis and to assess disease progression and re-activation in white-dot syndromes [10].

The gold standard for retinal imaging is fluorescein angiography (FA) [11].

However, indocyanine green angiography (ICGA) is probably the current best tool to indicate the true extent of choriocapillaropathies [12-14].

But all current imaging modalities concentrate on the retina, retinal pigment epithelium (RPE), or choroid. However, optical coherence tomography angiography (OCTA) is a new 3D non-invasive diagnostic method which is capable of examining the retinal and choroidal vasculature concurrently and allows for a detailed study of vascular perfusion in all layers via motion contrast imaging.

Until now, there are only case reports concerning OCTA and one case series encompassing 3 patients in SC [15]. Therefore, the aim of this study is to describe these findings in greater extent and compare them to known diagnostic tools, such as FA, ICGA, fundus autofluorescence (FAF), and enhanced depth imaging optical coherence tomography (EDI-OCT) [16-23].

\section{Methods \\ Patients}

This prospective study was approved by the local ethics committee (EA4/055/16) and adhered to the tenets of the declaration of Helsinki. Patients with SC were included between April and November 2016 after informed consent was given, so that their data could be used in this study as well as for the ability to perform a clinical examination and imaging. Diagnosis of SC was confirmed after exclusion of infectious diseases such as tuberculous uveitis [7].

\section{Clinical examination and imaging}

Full ophthalmologic examination, including snellen visual acuity test, Goldmann contact tonometry, slit-lamp examination, and fundoscopy with a 90-diopter lens, was performed and all patients received the following imaging modalities: EDI-OCT, FA, ICGA, and FAF. Visual acuity results were converted into a $\log$ MAR scale, according to the visual acuity measurement standard from the international council of ophthalmology.

All imaging modalities were carried out by an experienced ophthalmic photographer with the Heidelberg SPECTRALIS OCT, Heidelberg Engineering, Heidelberg, Germany (v1.9.2014.0 - Acquisition Module v6.4). The technical aspects of the machine were standardized as follows: for the EDI-OCT - Scan angle of $20^{\circ}, 512$ AScans for 19 Sections, inter-section distance of $250 \mu \mathrm{m}$, scaling of $11.5 \mu \mathrm{m} /$ pixel and an ART of 100 frames; for the FAF - Scan angle of $55^{\circ}, 768$ A-Scans, scaling of $20 \mu \mathrm{m} /$ pixel, and an ART of 100 frames.

FA and ICGA were performed. The early, intermediate and late phases of both imaging modalities were examined and the activity state was determined.

Inactive SC was identified based on clinical findings of fundoscopy and on multimodal imaging.

Active SC can lead to worsening of the vision, metamorphopsia, inflammation signs at the border of the previous lesions and neovascularization at the borders of the lesion. In FAF, active $\mathrm{SC}$ is described by images showing a peripheral hypoautofluorescence area surrounding the hyperautofluorescent borders of the lesions. Old atrophic areas are (because of the loss of choriocapillaris) completely hypoautofluorescent [2426]. In FA, active SC shows hypofluorescence surrounded by a hyperfluorescent border, with staining and leakage in the late phases. Inactive SC lesions are hypofluorescent at first, acquiring a hyperfluorescent edge later. ICGA shows hypofluorescent lesions throughout the entire examination, but usually of greater (and more reliable) extent in comparison to FA $[27,28]$.

\section{OCT angiography}

OCTA images were acquired using a prototype SPECTRALIS OCT device (SPECTRALIS ${ }^{\circ}$, Heidelberg Engineering, Heidelberg, Germany) using a prototype software (6.4.204.0) that applied an OCTA acquisition algorithm on which the now commercially released version is based. Images were acquired with an A-scan rate of 70, 000 per second and a $15^{\circ} \times 10^{\circ}$ scan angle protocol was used. A total of $261 \mathrm{~B}$-scans resulting in images with an axial resolution of approximately $4 \mu \mathrm{m}$, within B-scan resolution of approximately $11 \mu \mathrm{m}(6.99 \mu$ mpixel), and between B-scan resolution of also approximately $11 \mu \mathrm{m}$. The OCTA C-scan derived from the B-scans allows for $3 \mathrm{D}$ visualization of the different retinal and choroidal vascular plexuses. The scanning frame dimensions were $4.2 \times 2.8 \mathrm{~mm}$, being centered at the macula.

A standardized reproduction of the superficial capillary plexus (SCP - at $30 \mu \mathrm{m} \pm 30 \mu \mathrm{m}$ below the inner limiting membrane, ILM, representing the ganglion cell layer), deep capillary plexus (DCP - at $130 \mu \mathrm{m} \pm 12.5 \mu \mathrm{m}$ below 
the ILM, representing the inner nuclear layer), RPE, Choriocapillaris (at $10 \mu \mathrm{m} \pm 0 \mu \mathrm{m}$ below the Basement Membrane, BM), Sattler's Layer (at $70 \mu \mathrm{m} \pm 10 \mu \mathrm{m}$ below the BM,) and Haller's Layer (at $140 \mu \mathrm{m} \pm 10 \mu \mathrm{m}$ below the BM) were attempted based on the "Atlas OCT Angiography in AMD" [29]. Each OCTA produced by the software's algorithm was analyzed for the presence of the expected anatomy to be found at the respective depth of the retina, this being also based on the "Atlas OCT Angiography in AMD" [29]. For this comparison, interobserver correlation was assessed. The identification and differentiation between the Sattler's and Haller's layer were done with recourse to the following criteria: the Sattler's layer was identified by vessel-like entities in a hyper-intense grayish background, appearing below the choriocapillaris until reaching the Haller's layer, an area of hypo- and hyper-intense signals corresponding to bigger vessels.

Furthermore, the images were then evaluated with respect to the presence of segmentation errors, which were defined by clear deviations of the segmentation line from the observable path of the anatomical structures, due to imprecise or altogether lacking identification of the true reference point. Afterwards, the images were optimized to improve the analysis. Segmentation boundaries were manually changed to best reproduce the expected anatomy (mostly in the retinal pigment epithelium and below).

\section{Characterization of lesions}

The atrophic area at the level of the choriocapillaris and RPE were measured manually (in $\mathrm{mm}^{2}$ ) after proper and individual segmentation by ML and SM of the corresponding OCTA slab. This was achieved using the linear caliper tool of the OCTA embedded in the Heidelberg Eye Explorer Software (Viewing Module 6.6.0.1), in order to compare both measurements and reliability of the technology across observers.

\section{Statistics}

Statistical Analysis was performed using IBM SPSS Statistics v23. First, we analyzed the data with descriptive and frequency statistics. A t-Test-Paired Two-Sample Test assuming Equal Variances was employed to compare the area of atrophy at the choriocapillaris and RPE of each patient. A Mann-Whitney U Test was used to compare the best corrected visual acuity (BCVA) of patients with SE and patients without SE. A one-way ANOVA-Test was used to assess the relationship between the BCVA and the presence of SE on the OCTA's. Interobserver agreement for qualitative data was studied using Cohen's kappa and Lin's concordance correlation coefficient for quantitative data. The results were regarded as statistically significant if $p$ was below 0.05 [30].

\section{Results}

Patients

Twenty-four eyes of 12 patients with SC were examined at our department of Ophthalmology. Eight patients (67\%) were female and 4 male (33\%). The mean age of all patients on the date of data collection was $60+/-$ 15.6 standard deviation (SD) years.

One patient (2 eyes) was diagnosed with macular SC, 2 with atypical SC, and 9 with peripapillary SC. One eye was excluded due to the presence of significant macular edema and one due to the interruption of the examination, as requested by the patient owing to lightheadedness.

In total, 22 eyes of 12 patients with the diagnosis of currently inactive SC were examined and evaluated. Patient demographics and disease status are shown in more detail in Table 1.

The mean BCVA was 20/100 [0.71 logMAR (SD 0.96)] with a median of 20/36 [0.25 logMAR (range 0-3)]. Three patients (25\%) were under therapy with Cyclosporine A with a daily dose range of $1-3 \mathrm{mg} / \mathrm{kg}$. Two patients $(17 \%)$ were treated with Interferon alpha-2a (3 Mio. IE every 3 days) and another 2 patients (17\%) with Methotrexate (15 mg/week). Other therapies consisted of Azathioprine (75 mg/day), Adalimumab (40 mg s.c. every 2 weeks) in combination with corticosteroids (7.5 $\mathrm{mg} /$ day), and Methotrexate (7.5 mg/week) in combination with corticosteroids ( $7.5 \mathrm{mg} /$ day), each in one patient. One patient did not receive any therapy as shown in Table 1. Mean duration of disease was 5.8 years (SD 4.0) with a median of 5.9 years. Mean time since last recurrence was 4.5 years (SD 4.0) with a median of 3 years.

\section{Multimodal imaging}

On EDI-OCT, the lesions resembled geographic atrophy with RPE and choroidal thinning and loss of the ellipsoid portion of the inner segments (EPIS), and cones outer segment tips (COST) lines. Furthermore, atrophy of the choriocapillaris was observed in $100 \%$ $(22 / 22)$ of eyes and atrophy of the choriocapillaris and RPE in 91\% (20/22 eyes). Additional loss of the inner retinal layers was seen in more advanced stages of atrophy in 12/22 eyes (55\%) with increased visibility of the choroid, which was isoreflective in all patients (Fig. 1a).

In FAF, the lesions were hypoautofluorescent at all stages in $100 \%$ of eyes (Fig. 1b). In FA, the lesions were mostly hypofluorescent in the early phase with welldefined hyperfluorescent margins with no leakage nor blurry margins, whereas they remained hypofluorescent in ICGA, providing a better discernment of the true extent of the disease as shown in Fig. 1c. 
Table 1 Patients demographics

\begin{tabular}{|c|c|c|c|c|c|c|c|c|}
\hline $\begin{array}{l}\text { Age, } \\
\text { years }\end{array}$ & Sex & $\begin{array}{l}\text { Type of } \\
\text { Disease }\end{array}$ & $\begin{array}{l}\text { Visual Acuity } \\
\text { OD }\end{array}$ & $\begin{array}{l}\text { Visual Acuity } \\
\text { OS }\end{array}$ & $\begin{array}{l}\text { Activity } \\
\mathrm{OU}\end{array}$ & $\begin{array}{l}\text { Years since first } \\
\text { Visit }\end{array}$ & $\begin{array}{l}\text { Years since last } \\
\text { recurrence }\end{array}$ & Treatment OU \\
\hline 81 & M & A & $20 / 32$ & - & Inactive & 8 & 3 & Prednisolon \\
\hline 57 & W & A & $20 / 400$ & $20 / 60$ & Inactive & 2.5 & 2.5 & Methotrexate \\
\hline 47 & W & M & $20 / 125$ & 20/2000 & Inactive & 7 & 7 & Methotrexate \\
\hline 34 & W & $P$ & $20 / 20$ & $20 / 20$ & Inactive & 2.8 & 2.8 & Adalimumab \\
\hline 63 & W & P & $20 / 25$ & - & Inactive & 15.7 & 15.7 & Azathioprine \\
\hline 73 & M & $P$ & $20 / 200$ & $20 / 100$ & Inactive & 9 & 2 & Ciclosporine \\
\hline 34 & W & $P$ & $20 / 20$ & $20 / 50$ & Inactive & 1.4 & 1.4 & Ciclosporine \\
\hline 68 & W & $P$ & $20 / 25$ & $20 / 25$ & Inactive & 3.7 & 3.7 & Ciclosporine \\
\hline 54 & W & $P$ & $20 / 20$ & $20 / 20000$ & Inactive & 6 & 3 & Interferon- $a$ \\
\hline 68 & M & P & $20 / 20$ & $20 / 40$ & Inactive & 5.8 & 5.8 & Interferon-a \\
\hline 62 & M & P & $20 / 2000$ & $20 / 32$ & Inactive & 1.6 & 1.6 & $\begin{array}{l}\text { Methotrexate + } \\
\text { Prednisolon }\end{array}$ \\
\hline 79 & W & $P$ & $20 / 20000$ & $20 / 20$ & Inactive & 6 & 6 & - \\
\hline
\end{tabular}

$A$ atypical; $M$ macular; $P$ peripapillary; $O D$ right eye; $O S$ left eye; $O U$ both eyes

\section{OCTA}

Standardized representation of the different retinal and choroidal layers using OCTA showed that the SCP was identified correctly in 19 of 22 eyes (86\%), while Sattler's and Haller's layer could only be identified correctly in 10 eyes (45\%). DCP and RPE could be discerned in 15 eyes (68\%), and choriocapillaris in 14 eyes (64\%) (mean of interobserver agreement measurements: $\mathrm{k}=0.891$; range: $0.891-1 ; \mathrm{p}<0.001$ ) (Figs. 2 and 3).

When comparing the lesions in a multimodal imaging approach, the extent of the lesions remained constant throughout FA/ICGA/FAF/EDI-OCT/OCT, with more details of the deeper layers being offered by OCTA.

The mean area of focal flow impairment and atrophy was, respectively, $2.64 \mathrm{~mm}^{2} \quad\left(\mathrm{SD}=3.37 \mathrm{~mm}^{2}\right)$ in the

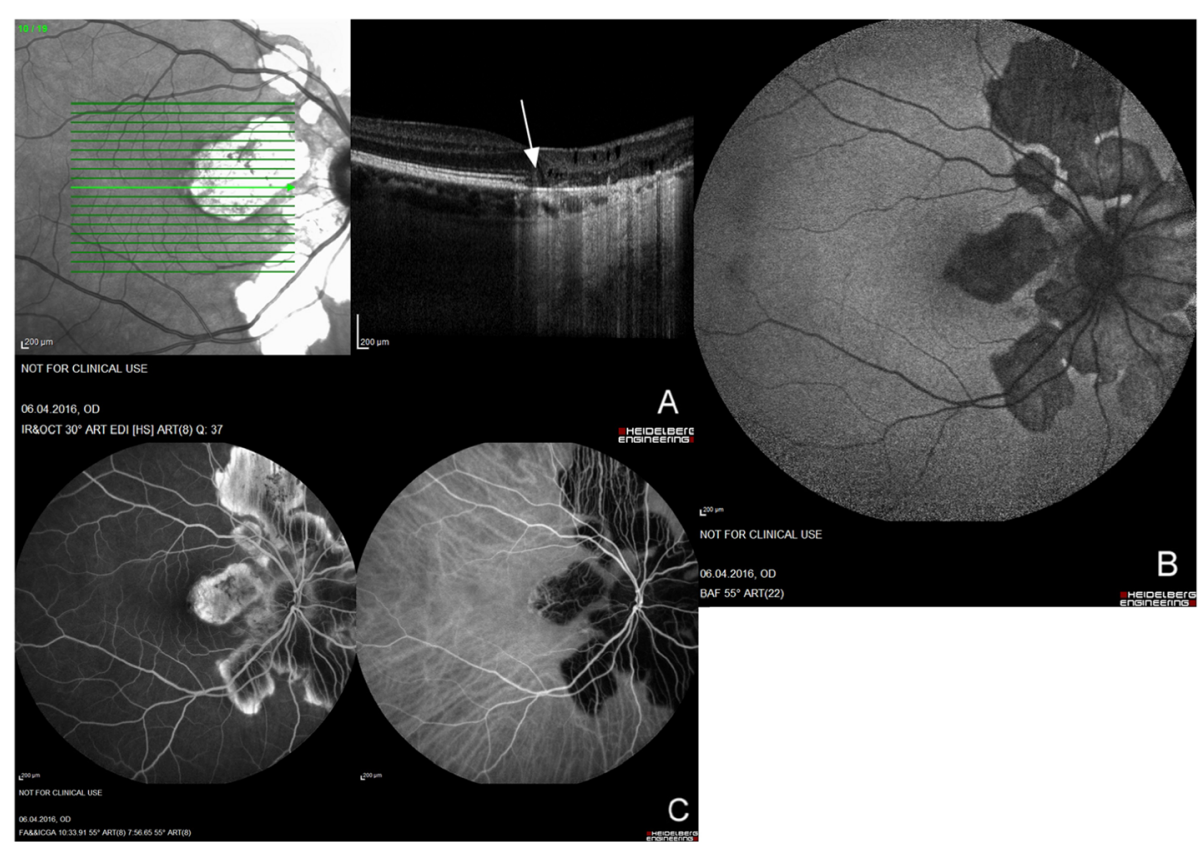

Fig. 1 Multimodal imaging of a 53-year-old female patient with peripapillary Serpiginous Choroiditis, showing the clear loss of choriocapillaris/ RPE/EPIS/COST nasal to the fovea (arrow) and the centrifugal progression of the disease from the optic disc. Representations with an angle of $30^{\circ}$ of SD-EDI-OCT of the macula (A), FAF (B), and FA/ICGA (C) 


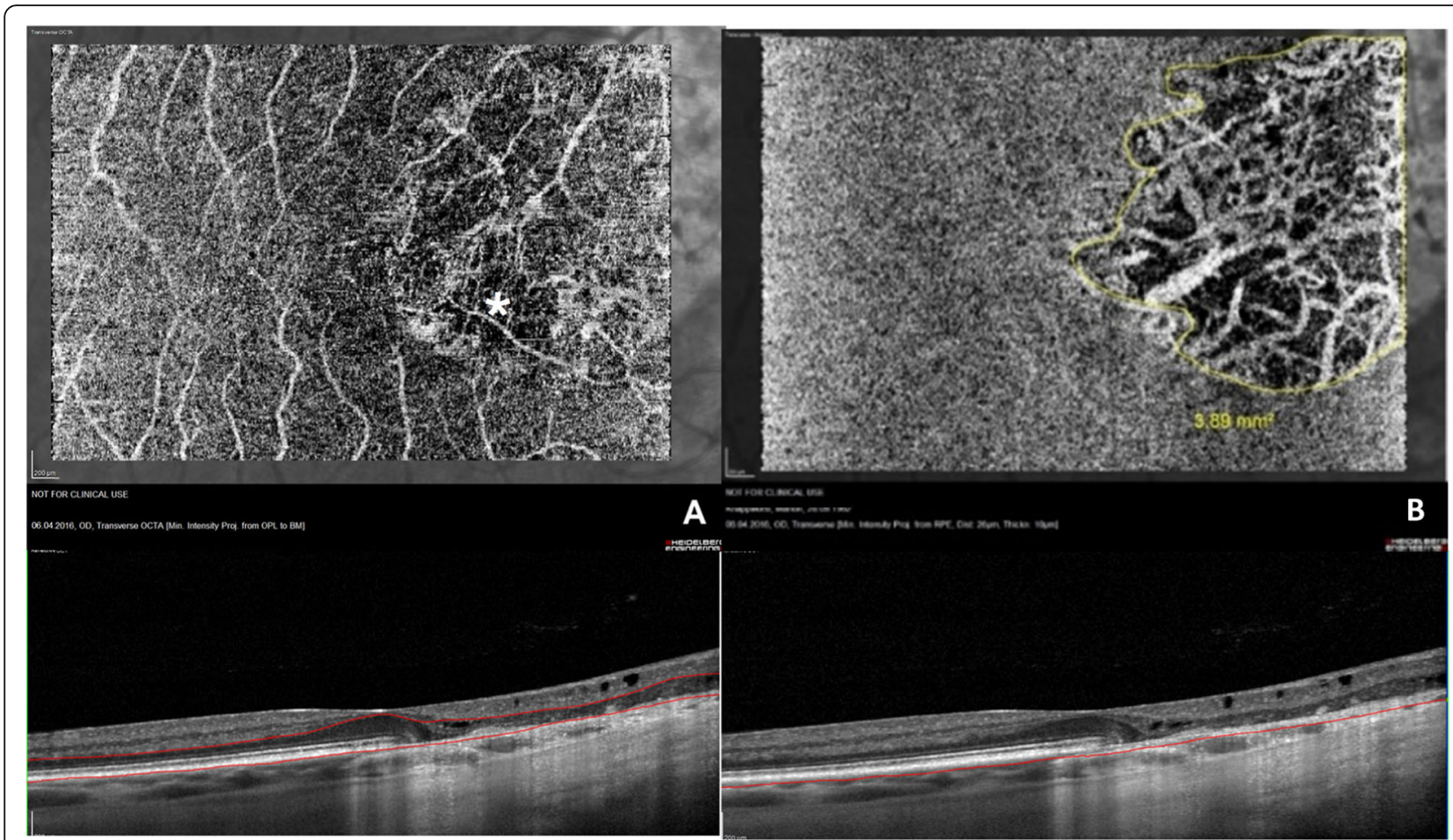

Fig. 2 Choroidal changes in SC. Segmentation of the retina showing a defect (asterisk) of the RPE and choriocapillaris (a), and just the choriocapillaris in greater detail (b). The OCT angiograms are projections of the retina between the automatically segmented outer plexiform layer and the manually corrected basal membrane in (a) and at an imaginary line running $10 \mu \mathrm{m}$ below and parallel to the manually segmented retinal pigment epithelium in (b), shown in the corresponding OCT B-Scans below

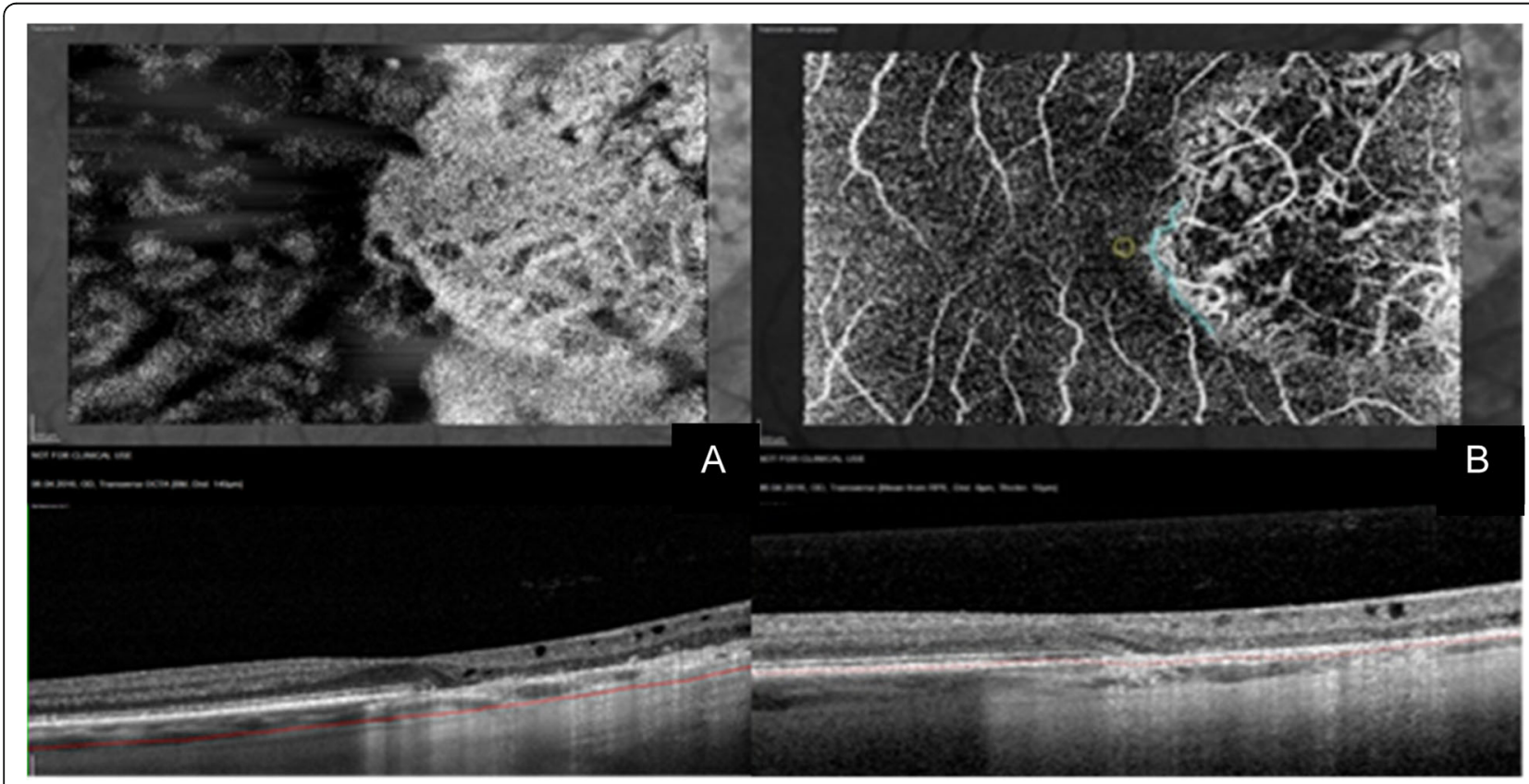

Fig. 3 Choroidal changes in SC (continued). The hypoperfusion seen in Fig. 2 leads to a window defect responsible for the "White on Black effect" in the Haller's Layer seen here at the top of (a). $\mathbf{b}$ displays the same defect at the level of the retinal pigment epithelium, showing that the area corresponding to the foveola was spared. The OCT angiograms are projections of the retina at an imaginary line running $140 \mu \mathrm{m}$ inferiorly and parallel to the basal membrane in (a) and through the manually segmented retinal pigment epithelium in (b), shown in the corresponding OCT B-Scans below 
choriocapillaris and $2.36 \mathrm{~mm}^{2}\left(\mathrm{SD}=3.33 \mathrm{~mm}^{2}\right)$ in the RPE. This showed no statistically significant difference $(\mathrm{p}=0.39)$ and a high degree of inter observer agreement (Lin's concordance correlation coefficient $=0.867$; CI: $0.71-0.94)$. The area of choriocapillaris hypoflow was only smaller than the area of atrophy in the RPE in one eye $\left(9.37 \mathrm{~mm}^{2}\right.$ to $10.32 \mathrm{~mm}^{2}$, respectively).

The atrophy caused by SC led to the presence of white-on-black effect in the Haller's layer, this referring to a large white area on a black background corresponding grossly to the atrophic area. This is shown in Fig. 3a. There were no observable differences between the 3 forms of SC.

Both figures (Fig. 3a and b) pertain to the same patient, clearly showing that the foveola was spared when viewed on the OCT of Fig. 3a and on the OCTA of Fig. 3b, which is the reason for a BCVA of 20/20 [0.1 logMAR] on examination, even though the vision had been 6/38 [0.8 logMAR] during a recent active inflammatory episode, which was successfully treated with Interferon- $\alpha$.

OCTA images of 10 eyes (46\%) showed significant SE. Mean BCVA of eyes with SE's was 20/400 [1.3 logMAR] in contrast to the patients' group without SE's with a BCVA of 20/34 [0.23 logMAR] (Mann-Whitney U Test: $\mathrm{z}=-2.15, \mathrm{p}=0.031)$. SE's were only seen in 3 eyes $(14 \%)$ with a BCVA better than 20/100 [0.7 logMAR]. A statistically significant "correlation"/relationship was established between low BCVA and increased presence of SE's using a one-way ANOVA $(\eta=0.57, \mathrm{~F}=9.76, \mathrm{p}=0.005)$.

\section{Discussion}

OCTA is a non-invasive diagnostic method of assessing the retinal and choroidal vascular layers. It is currently suggested that the corollary atrophy of the choriocapillaris resulting from its hypoperfusion, as first reported as a loss of choriocapillary homogeneity on OCTA by Khan et al., causes the consecutive destruction of RPE and photoreceptors which leads to vision loss [21, 23].

In our study, OCTA images showed a clear hypoperfusion of the choriocapillaris in $100 \%$ of our patients, which compared well to the atrophic areas observed on the EDI-OCT [22].

Equally, the atrophic areas in the RPE represented by hypoperfusion in the OCTA, greatly and reliably corresponded to the same atrophic areas seen in FA, ICGA and FAF. However, the extent of choriocapillaris hypoperfusion (mean area $=2.64 \mathrm{~mm}^{2}$ ) was greater than the atrophic areas of the RPE (mean area $=$ and $2.36 \mathrm{~mm}^{2}$ ) in all but one eye, which could be explained by the choroidal origin of the disease, even though this difference was not statistically significant. These same areas were hypoautofluorescent in all eyes, since the disease was inactive. According to the literature, hypoautofluorescent lesions with hyperautofluorescent borders were to be expected in active disease [21-23]. The study of Montorio et al. found a complete loss of detectable flow in 2 active eyes with $\mathrm{SC}$, whereas choroidal vessel rarefaction and loss of choriocapillaris could be seen in inactive eyes (20 eyes) [31].

The pathophysiology of the 3 different forms of SC remains unclear. No differences could be seen between these forms in all imaging modalities and none have been described to date.

Although OCTA proved to be useful for imaging of the choriocapillaris, the representation of the deeper choroidal layers contained challenges. The Sattler's layer examination did not reveal much information, whereas a conversion from a "black-on-white" effect to a "white-on-black" effect could be seen in the Haller's layer below the atrophic regions, as demonstrated in the literature. This effect remains to be explained [19]. Current theories explain it as a consequence of the physics of wave propagation through the different retinal and choroidal layers, in that the propagation through the atrophic lesions is not hampered by so many layers/cells as it is in the neighbor regions, causing this "white-on-black" effect much later if at all. Another hypothesis is that this effect can be a consequence of the absence of or different flow (e.g. too slow or too fast) or characteristics of the vessels and surrounding tissues within these regions due to inflammation as first proposed by Montorio et al. [31].

The standard adjustments of the retinal and choroidal layers did not correspond very well with the structures of deeper layers.

SE's were the main problem in patients with macular edema or scarring so that the interpretation of these images was difficult and led to the exclusion of one eye. Statistically significant differences of mean BCVA could be seen between patients with and without SE's which may be due to a missing fixation of the patients and a longer examination time of OCTA. The newer software with faster examination time and auto-fixation program overcomes some of these problems.

One last disadvantage concerned the lack of detection of fluid dynamics (pooling, staining, leakage) even though one could argue that this provided in-depthselective images, free of masking affects.

Although this study's conclusions are limited by the numbers of eyes evaluated and there is missing knowledge of the pathophysiology of $\mathrm{SC}$, lesions presenting in the disease can be visualized using OCTA as reliably as through the currently established techniques (ICGA, FA and FAF). The use of OCTA offers a non-invasive and high-resolution means to assess the disease in three dimensions, allowing for the ability to precisely locate the atrophic area. Furthermore, OCTA may help determine if the disease is in an active phase via, for example, identification of $\mathrm{CNV}$ (a known complication of this and 
other entities) even when ICGA is not able to do so, as reported by Mandadi et al. and as observed in tuberculous serpiginous-like Choroiditis. In this study, it was not possible to prove this hypothesis as all enrolled patients were in inactive disease stages [32].

Additionally, analogies could be seen between the changes of the choriocapillaris and choroid caused by SC and geographic atrophy. These included a focal flow impairment and rarefaction in the choriocapillaris beneath the area of the atrophic lesions, which is argued to represent non-perfused or hypo-perfused choroidal vessels with non-detectable flow in the literature [33-35].

OCTA is clinically valuable in the management of patients with SC. Even though the representation of the lesions with OCTA corresponded to the ones seen in the FA/ICGA/FAF/EDI-OCT, the inclusion of this tool in a multimodal approach for the diagnosis and management of SC should be considered as an extra or alternative examination.

\section{Conclusions}

OCTA is able to assess vascular lesions of the choroid and retina in patients with serpiginous Choroiditis with a high degree of correlation to other diagnostic modalities.

\section{Abbreviations \\ BCVA: Best corrected visual acuity; BM: Basement membrane; CNV: Choroidal neovascularisation; COST: Cones outer segment tips; DCP: Deep capillary plexus; EDI-OCT: Enhanced depth imaging optical coherence tomography; EPIS: Ellipsoid portion of the inner segments; FA: Fluorescein angiography; FAF: Fundusautofluorescence; ICGA: Indocyanine green Angiography; ILM: Inner limiting membrane; OCTA: Optical coherence tomography angiography; RPE: Retinal pigment epithelium; SC: Serpiginous choroiditis; SCP: Superficial capillary plexus; SD: Standard deviation; SE: Segmentation error}

\section{Acknowledgements}

We thank DS for performing the different imaging modalities and AT for proofreading.

\section{Authors' contributions \\ SM prepared the manuscript and analyzed and interpreted the data with a contribution from ML. DP helped with the ethics approval and study preparation. UP assisted in selecting the patients and confirming their diagnosis. AMJ contributed in writing and reviewing the manuscript. SW was responsible for the development and organization of the study and was a major contributor in writing the manuscript. All authors read and approved the final manuscript.}

\section{Funding}

We acknowledge support from the German Research Foundation (DFG) and the Open Access Publication Fund of Charité - Universitätsmedizin Berlin with regards to the funding of publication fees. Their support played no role in the design of the study and collection, analysis, and interpretation of data and in writing the manuscript.

\section{Availability of data and materials}

Data and materials related to this work are available from the corresponding author upon reasonable request.

\section{Ethics approval and consent to participate}

This prospective study was approved by the ethics committee of the Charite - Universitätsmedizin Berlin (EA4/055/16) and adhered to the tenets of the Declaration of Helsinki. Written informed consent was obtained from all patients.

\section{Consent for publication}

Patients' anonymized data was used after informed written consent was given

All patients signed a written consent for the use of their clinical data and images in this study.

\section{Competing interests}

AMJ has served as principal investigator or consultant for: Allergan, Novartis, Bayer, Heidelberg engineering. UP has served as principal investigator or consultant for: Abbvie, Alcon, Allergan, Novartis, Santen, Thea. SW has served as consultant for: Allergan, Novartis, Bayer, MSD, Heidelberg engineering. DP has served as consultant for: Allergan, Heidelberg engineering. The remaining authors declare that they have no competing interests.

Received: 8 January 2020 Accepted: 18 June 2020

Published online: 30 June 2020

\section{References}

1. Ezra DB, Forrester JV. Fundal white dots: the spectrum of a similar pathological process. Br J Ophthalmol. 1995;79:856-60.

2. White spot syndromes of the retina: a hypothesis based on the common genetic hypothesis of autoimmune/inflammatory disease. Am J Ophthalmol. 2003:135(3):376-9.

3. Quillen DA, Davis JB, Gottlieb JL, et al. The white dot syndromes. Am J Ophthalmol. 2004:137(3):538-50

4. Thurau S, Pleyer U. Entzündliche Augenerkrankungen. 1st ed. Heidelberg: Springer; 2014.

5. Hardy RA, Schatz H. Macular geographic helicoid choroidopathy. Arch Opthalmol. 1987;105(9):1237-42.

6. Cunningham ET Jr, Gupta A, Zierhut M. The creeping Choroiditides Serpiginous and multifocal Serpiginoid Choroiditis. Ocul Immunol Inflamm. 2014;22(5):345-8.

7. Mackensen F, Becker MD, Wiehler U, Max R, Dalpke A, Zimmermann S QuantiFERON TB-gold - a new test strengthening long-suspected Tuberculous involvement in Serpiginous-like Choroiditis. Am J Ophthalmol. 2008:146:761-6.

8. Christmas NJ, Oh KT, Oh DM, Folk JC. Long-term follow-up of patients with Serpinginous Choroiditis. Retina. 2002;22:550-6.

9. Sobaci G, Bayraktar Z, Bayer A. Interferon alpha-2a treatment for serpiginous choroiditis. Ocul Immunol Inflamm. 2005;13(1):59-66.

10. Knickelbein JE, Sen HN. Multimodal Imaging of the White Dot Syndromes and Related Diseases. J Clin Exp Ophthalmol. 2016;7(3):570.

11. Vabres G. Study of an atypical unilateral choriodopahty of the posterior pole with centrifugal serpiginous development (clinical, angiofluorographic and functional aspects). Ann Ocul. 1972;205(8):927-39.

12. Bouchenaki N, Cimino L, Auer C, et al. Assessment and classification of choridal vasculitis in posterior uveitis using indocyanine green angiography. Klin Monatsbl Augenheilkd. 2002;219(4):243-9.

13. Giovannini A, Ripa E, Scassellati-Sforzolini B, et al. Indocyanine green angiography in serpiginous choroidopathy. Eur J Ophthalmol. 1996;6(3): 299-306.

14. Squirrell DM, Bhola RM, Talbot JF. Indocyanine green angiographic findings in serpiginous choroidopathy: evidence of a widespread choriocapillaris defect of the peripapillary area and posterior pole. Eye (Lond). 2001;15:336-8.

15. Desai R, Nesper P, Goldstein DA, Fawzi AA, Jampol LM, Gill M. OCT Angiography Imaging in Serpiginous Choroidopathy. Ophthalmol Retina. 2018;2(4):351-359.

16. Carreño E, Portero A, Herreras JM, López Ml. Assesment of fundus autofluorescence in serpiginous and serpiginous-like choriodopathy. Eye (Lond). 2012;26(9):1232-6.

17. Piccolino FC, Grosso A, Savini E. Fundus autofluorescence in serpiginous choroiditis. Graefes Arch Clin Exp Ophthalmol. 2009;247:179-85.

18. Invernizzi A, Agarwal A, Cozzi M, Viola F, Nguyen QD, Staurenghi G. Enhanced depth imaging optical coherence tomography features in areas of choriocapillaris hypoperfusion. Retina. 2016;36(10):2013-21.

19. Fang PP, Harmening WM, Müller PL, Lindner M, Krohne TU, Holz FG. Technical principles of OCT angiography. Ophthalmologe. 2016;113:6-13.

20. Choi W, Mohler KJ, Potsaid B, et al. Choriocapillaris and choroidal microvasculature imaging with ultrahigh speed OCT angiography. PLoS One. 2013;8(12):e81499 doi: 10.137. 
21. Khan HA, Shahzad MA. Multimodal imaging of Serpiginous Choroiditis. Optom Vis Sci. 2017 Feb;94(2):265-9.

22. El-Ameen A, Herbort CP Jr. Serpiginous Choroiditis imaged by optical coherence tomography. Retin Cases Brief Rep. 2018;12(4):279-85.

23. Seong JA, Seung HP, Byung RL. Multimodal Imaging Including Optical Coherence Tomography Angiography in Serpiginous Choroiditis. Ocular Immunol Inflamm. 2017;25(2):287-29.

24. Arantes TE, Matos K, Garcia CR, et al. Fundus autofluorescence and spectral domain optical coherence tomography in recurrent serpiginous choroiditis: case report. Ocul Immunol Inflamm. 2011;19(1):39-41.

25. Cardillo Piccolino F, Grosso A, Savini E. Fundus autofluorescence in serpiginous choroiditis. Graefes Arch Clin Exp Ophthalmol. 2009;247(2):179-85.

26. Carreño E, Portero A, Herreras JM, López Ml. Assesment of fundus autofluorescence in serpiginous and serpiginous-like choroidopathy. Eye (Lond). 2012;26(9):1232-6. https://doi.org/10.1038/eye.2012.121.

27. Giovannini A, Mariotti C, Ripa E, Scassellati-Sforzolini B. Indocyanine green angiographic findings in serpiginous choroidopathy. Br J Ophthalmol. 1996; 80(6):536-40. https://doi.org/10.1136/bjo.80.6.536.

28. Squirrell $D$, Bhola R, Talbot J. Indocyanine green angiographie findings in serpiginous choroidopathy: evidence of a widespread choriocapillaris defect of the peripapillary area and posterior pole. Eye. 2001;15:336-8. https://doi. org/10.1038/eye.2001.110.

29. Coscas G, Lupidi M, Coscas F. Atlas OCTAngiography in AMD, comparison with multimodal imaging; 2015.

30. Holladay JT. Proper Method for Calculating Average Visual Acuity. J Refract Surg. 1997;13:389-391 - (Special article).

31. Montorio, et al. Swept-source optical coherence tomography angiography in serpiginous choroiditis. Br J Ophthalmol. 2018;102(7):991-5.

32. Mandadi SKR. Novel findings on optical coherence tomography angiography in patients with tubercular serpiginous-like choroiditis. Retina. 2017;37(9):1647-59.

33. Moult EM, Waheed NK, Novais EA, et al. Swept-source optical coherence tomography angiography reveals Choriocapillaris alterations in eyes with nascent geographic atrophy and drusen-associated geographic atrophy. Retina. 2016;36(Suppl 1):S2-S11.

34. Corbelli E, Sacconi R, De Vitis LA, et al. Choroidal round Hyporeflectivities in geographic atrophy. PLOS ONE 2016;11(11): e0166968.

35. Kvanta A, Casselholm de Salles M, Amrén U, Bartuma H. Optical coherence tomography angiography in geographic atrophy. Retina. 2017;37(5):936-42.

\section{Publisher's Note}

Springer Nature remains neutral with regard to jurisdictional claims in published maps and institutional affiliations.

Ready to submit your research? Choose BMC and benefit from:

- fast, convenient online submission

- thorough peer review by experienced researchers in your field

- rapid publication on acceptance

- support for research data, including large and complex data types

- gold Open Access which fosters wider collaboration and increased citations

- maximum visibility for your research: over $100 \mathrm{M}$ website views per year

At $\mathrm{BMC}$, research is always in progress.

Learn more biomedcentral.com/submissions 